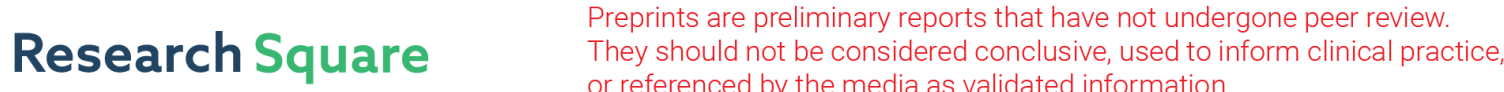 \\ Glucose feeds the TCA cycle via environmental ethanol in fermenting yeast
}

\section{Tianxia Xiao}

Princeton University

Artem Khan

The Rockefeller University https://orcid.org/0000-0002-1240-8674

\section{Yihui Shen}

Princeton University

\section{Li Chen}

Fudan University

Joshua Rabinowitz ( $\nabla$ joshr@Princeton.EDU)

Princeton University https://orcid.org/0000-0002-1247-4727

\section{Article}

Keywords:

Posted Date: December 28th, 2021

DOI: https://doi.org/10.21203/rs.3.rs-1162391/v1

License: (c) (1) This work is licensed under a Creative Commons Attribution 4.0 International License. Read Full License

Version of Record: A version of this preprint was published at Nature Chemical Biology on August 15th, 2022. See the published version at https://doi.org/10.1038/s41589-022-01091-7. 


\section{Glucose feeds the TCA cycle via environmental ethanol in 2 fermenting yeast}

\section{Authors}

4 Tianxia Xiao ${ }^{1}$, Artem Khan ${ }^{1,3}$, Yihui Shen², Li Chen²,4, Joshua Rabinowitz ${ }^{1,2}$ ( $\left.^{*}\right)$

$5{ }^{1}$ Lewis Sigler Institute for Integ rative Genomics and Department of Chemistry, Princeton University, Washington

6 Road, Princeton, NJ 08544, USA

$7 \quad 2$ Lewis-Sigler Institute for Integ rative Genomics, Princeton University, Princeton, NJ, 08544, USA.

83 The David Rockefeller Graduate Program in Bioscience, The Rockefeller University, New York, NY, USA.

$9{ }^{4}$ Shanghai Key Laboratory of Metabolic Remodeling and Health, Institute of Metabolism \& Integrative Biology,

10 Fudan University, Shanghai, 200433, China.

\section{Abstract}

Ethanol and lactate are typical waste products of glucose fermentation. In mammals, glucose is catabolized by glycolysis into circulating lactate, which is broadly used throughout the body as a carbohydrate fuel. Individual cells can both uptake and excrete lactate, uncoupling glycolysis from glucose oxidation. Here we show that similar uncoupling occurs in the yeast Saccharomyces cerevisiae. Even in fermenting yeast that are net releasing ethanol, media ${ }^{13} \mathrm{C}$-ethanol rapid enters and is oxidized to acetaldehyde and acetyl-CoA. This is evident in exogenous ethanol being a major source of both cytosolic and mitochondrial acetyl units. ${ }^{2} \mathrm{H}$-tracing reveals that ethanol is also a major source of both $\mathrm{NADH}$ and $\mathrm{NADPH}$, and this role is augmented under oxidative stress conditions. Thus, uncoupling of glycolysis from the oxidation of glucose-derived carbon via rapid reversible reactions is an ancient and conserved feature of eukaryotic metabolism.

\section{Introduction}

Fermentation occurs widely across Kingdoms, converting glucose into organic waste products ${ }^{1-4}$. In mammals, the main such product is lactate. Until recently, it was commonly assumed that the liver and kidney were special in their capacity to clear circulating lactate, reconverting the waste (lactate) into fuel (glucose). New evidence suggests, however, that most mammalian tissues take up circulating lactate and oxidize it via the TCA cycle 5 . Indeed, it seems that most carbohydrate oxidation in mammals, rather than occurring by a tissue taking up glucose and fully oxidizing it to carbon dioxide, instead involves carbon flowing through circulating lactate as a metabolic intermediate. Thus, glycolysis is uncoupled from the TCA cycle via cellular uptake and/or excretion of lactate 5 . Biochemically, this occurs through the rapid reversible reactions linking intracellular pyruvate, via lactate dehydrogenase and monocarboxylate transporters, to circulating lactate.

Baker's yeast (Saccharomyces cerevisiae) is a prototypical fermentative unicellular organism ${ }^{6}$. Its rapid catabolism of glucose into ethanol $+\mathrm{CO}_{2}$ plays a central role in human society, contributing to such diverse fields as baking, beverages, and biofuels. S. cerevisiae is capable of growing aerobically on substrates 
including galactose, glycerol, and ethanol. But when provided with ample glucose, it will ferment even in the presence of adequate oxygen ${ }^{7}$. When glucose runs out, after a delay to rewire metabolism, aerobic growth will resume (the diauxic shift) ${ }^{8}$. As glucose fermentation in yeast parallels aerobic glycolysis in mammals, we were curious whether it similarly involves reversible excretion and uptake of the "waste product" (ethanol), rather than unidirectional waste elimination. We further wondered whether any such ethanol uptake during net fermentative metabolism might contribute to yeast's metabolic robustness.

Understanding these questions is relevant both for basic science and for bioengineering (e.g. as ethanol uptake might sometimes be disadvantageous). With these motivations in mind, we show that, even when fermenting, yeast actively exchanges environmental ethanol for intracellular acetaldehyde at a sufficiently rapid rate that intracellular acetyl units come substantially from environmental ethanol rather than directly from glucose. Moreover, such exchange enables ethanol to be a major source of NADH and NADPH, especially under oxidative stress conditions.

\section{Results}

\section{Fermenting yeast assimilate environmental ethanol}

Ethanol can enter and exit cells via simple diffusion ${ }^{9}$. Thus, exogenous ethanol may enter yeast, even if they are simultaneously excreting ethanol made internally from glucose. To differentiate two-carbon (2C) units from environmental ethanol versus internal glucose catabolism, we grew yeasts in typical minimal media (yeast nitrogen base, aerated, $30^{\circ} \mathrm{C}$ ) with unlabeled glucose until mid-exponential phase. We then pelleted the cells and resuspended them in yeast nitrogen base containing both glucose and ethanol, whose isotopic composition is under experimental control. The glucose and ethanol concentrations in the resuspension media were selected to approximate those naturally occurring during mid-exponential yeast growth in yeast nitrogen base with glucose as the carbon source (recognizing that by mid-exponential phase yeast will have converted a substantial amount of glucose into ethanol). Specifically, we provided glucose and ethanol at either equimolar concentrations ( $42 \mathrm{mM}$ each) or 1:1 mixture based on number of carbon atoms ( $28 \mathrm{mM}$ glucose and $84 \mathrm{mM}$ ethanol, "equicarbon") (Figure 1A).

We used ${ }^{13} \mathrm{C}$ NMR to measure rates of glucose uptake ( $f_{\text {gluc_up}}$ ) and conversion to ethanol via pyruvate decarboxylase $\left(f_{p d c}\right)$ from the cultures with $\left[U-{ }^{13} \mathrm{C}\right]$ glucose and unlabeled ethanol (Figure 1B). The rates measured by ${ }^{13} \mathrm{CNMR}$ ( $f_{\text {gluc_up }}$ and $\mathrm{f}_{\mathrm{pdc}}$ ) are similar among strains of $S$. cerevisiae with different respiratory capacity (FY4 and CEN.PK) and media substrate ratios (equimolar or equicarbon) (Supplementary figure 1A). In parallel, net ethanol flux was measured by ${ }^{1} \mathrm{H}$ NMR, revealing active fermentation (i.e. net ethanol excretion) (Figure 1C).

The yeast ethanol assimilation pathway involves oxidation of ethanol to acetate, which is converted into cytosolic acetyl-CoA by acetyl-CoA synthetases ${ }^{10}$. In attempt to directly trace potential ethanol uptake and utilization, we directly measured cellular acetyl-CoA labeling distributions by LC-MS in yeasts grown with unlabeled glucose and labeled ethanol (Figure 1D), finding substantial labeling (more than 50\%) from 
environmental ethanol (Figure 1E). This high [M+2] labeled fraction of acetyl-CoA is consistent across strains and media compositions (Supplementary Figure 1B).

We built a ${ }^{13} \mathrm{C}$ metabolic flux model to estimate the reversibility of the ethanol assimilation pathway (Figure $1 \mathrm{~F}$ ). The model was constrained by the measured glucose uptake rate, the net ethanol excretion rate, PDC flux (the flux representing gross glucose conversion to ethanol), and acetyl-CoA labeling from [U- $\left.{ }^{13} \mathrm{C}\right]$ ethanol. The model confirmed low PDH and high PDC flux, as typical for fermenting yeast (Figure 1G). Notably, it revealed a fast exchange flux between ethanol and acetaldehyde, with ethanol a major source of acetaldehyde even though net flux is in the direction of ethanol excretion (Figure 1G). This rapid exchange flux explains the substantial acetyl-CoA labeling from environmental ethanol (Figure 1D).

\section{Environmental ethanol contributes to fatty acid synthesis}

Acetyl-CoA exists as discrete cytosolic and mitochondrial pools. Fatty acid synthesis utilizes cytosolic acetylCoA (Figure 2A), thus fatty acid labeling selectively represents cytosolic acetyl-CoA labeling. In yeast fed either the equimolar or equicarbon mixture of unlabeled glucose and $\left[\mathrm{U}-{ }^{13} \mathrm{C}\right]$ ethanol, a majority of the carbon in newly synthesized fatty acids (i.e. those containing at least some label) was from environmental ethanol (Figure 2B, Supplementary Figure 2).

To quantify fraction of cytosolic acetyl-CoA coming from environmental ethanol, we fit the observed fatty acid mass isotope distribution to a binomial, reflecting the fact that each $2 \mathrm{C}$ unit incorporated into fat is selected stochastically, with the assumption that only labeled fatty acids are newly synthesized. Such fitting revealed around $60 \%$ environmental ethanol contribution (Figure $2 \mathrm{C}$ ). Thus, rather than being derived mainly internally by glycolysis and subsequent pyruvate catabolism, when environmental ethanol is present, cytosolic acetylCoA in Baker's yeast also comes from ethanol.

\section{Environmental ethanol supplies mitochondrial acetyl-CoA}

Formation of cytosolic acetyl-CoA from acetate is catalyzed by acetyl-CoA synthetases ${ }^{11,12}$. Such synthetases are not known in mitochondria. Accordingly, we were curious whether environmental ethanol could also contribute to mitochondrial acetyl-CoA. To this end, using the same tracing strategy as above, we examined whether environmental ethanol would label a metabolic product that is produced mitochondrially from acetylCoA, N-acetylglutamate (NAG), an intermediate in the arginine biosynthesis pathway (Figure 3A). In yeast fed either equimolar or equicarbon unlabeled glucose and [U-13C]ethanol, NAG was labeled both $[M+2]$ and $[M+4]$ while glutamate was only labeled [M+2] (Figure 3B, supplementary figure 3). To quantitate the fraction of mitochondrial acetyl-CoA coming from environmental ethanol, we inferred mitochondrial acetyl-CoA labeling from the observed mass isotope distribution of NAG and glutamate. The calculated [M+2] fraction of mitochondrial acetyl-CoA is around $60 \%$ (Figure $3 \mathrm{C}$ ), similar to cytosolic acetyl-CoA. Thus, environmental ethanol is a major source of both cytosolic and mitochondrial acetyl-CoA.

The enzyme succinyl-CoA acetate CoA transferase (Ach1) has been proposed as a potential means of generating mitochondrial acetyl-CoA from acetate, but it is physiological role has remained unproven ${ }^{13}$. Ach1 
deletion completely abolished [M+4] NAG (Figure 3B, supplementary figure 3), with the inferred mitochondrial acetyl-CoA labeling zero in this deletion strain. Notably, the deletion strain nevertheless has similar whole cell $[\mathrm{M}+2]$ acetyl-CoA labeling from ethanol (supplementary figure 4), implying that only a small fraction of total cellular acetyl-CoA is mitochondrial, with Ach1 the key mitochondrial acetate assimilation enzyme.

Mitochondrial acetyl-CoA contributes to the TCA cycle via citrate synthase (Figure 4A). Consistent with this, from the ${ }^{13} \mathrm{C}_{2}$-ethanol tracing, we observed [M+2] (iso)citrate, aconitate, $\alpha$-ketoglutarate, and succinate (Figure 4B, supplementary figure 5). Fumarate, malate, and aspartate (whose carbon skeleton comes from oxaloacetate) remained, however, largely unlabeled (Figure 4C, supplementary figure 5 ). The extensive labeling of succinate with limited labeling of fumarate or oxaloacetate pinpoints succinate dehydrogenase (complex II in the electron transport chain) as being functionally blocked during yeast fermentative growth ${ }^{14}$. Instead of being made by TCA turning, oxaloacetate and malate are generated by pyruvate carboxylase, using pyruvate made from glucose. Nevertheless, acetate from environmental ethanol is assimilated into the TCA cycle and drives conversion of these four-carbon TCA intermediates into citrate, $\alpha$-ketoglutarate, and $\alpha$ ketoglutarate's amino acid products.

\section{Acetaldehyde oxidation feeds NADPH}

Oxidation of ethanol to acetaldehyde generates NADH. Further oxidation of acetaldehyde into acetate via aldehyde dehydrogenase generates NADPH. When the canonical main NAPDH-production pathway, the oxidative pentose phosphate pathway, is deleted, the acetaldehyde dehydrogenase Ald6 is essential for yeast growth ${ }^{15}$. We were curious about the extent to which acetaldehyde derived from environmental ethanol contributes to NADH or NADPH. To explore this, we transferred fermenting yeast into glucose:ethanol as above, with either the glucose or the ethanol deuterium labeled. Specifically, we compared NADH and NADPH labeling from $[1-2 \mathrm{H}]$ glucose (the labeled hydrogen is transferred to NADPH via G6PD, encoded by gene zwf1) and $\left[1,1-{ }^{2} \mathrm{H}_{2}\right]$ ethanol (the labeled hydrogen is transferred to $\mathrm{NADH}$ by ADH and to NADPH via Ald) (Figure $5 A, D)$. Direct measurement of ${ }^{2} \mathrm{H}$-labeling in NADH and NADPH is technically challenging due to limited abundance and stability, but nevertheless we observed clear isotope shifts upon exposure to the ${ }^{2} \mathrm{H}$-labeled substrates, confirming contribution from glucose's position 1 hydrogen to NADPH and from ethanol's position 1 hydrogens to both NADH and NADPH (Supplementary Figure 5B,C,E,F).

To obtain more precise and compartment-specific information, we used fatty acid labeling to read out cytosolic NADPH labeling. Fatty acid synthesis incorporates 2 NADPH hydrides per acetyl group (Figure 5A) ${ }^{16}$. Strikingly, we observed greater deuterium labeling of fatty acids from $\left[1,1-{ }^{2} \mathrm{H}_{2}\right]$ ethanol than from $\left[1-{ }^{2} \mathrm{H}\right]$ glucose (Supplementary figure 7,8). This reflects a major contribution of ethanol-derived acetaldehyde to cytosolic NADPH via Ald6 (Figure 5B).

To convert the observed labeling into quantitative contributions to NADPH, we need to account for deuterium loss from NADPH via hydrogen-deuterium exchange with water mediated by flavin enzymes ${ }^{16,17}$. Experiments culturing cells in $\mathrm{D}_{2} \mathrm{O}$ revealed that about half of cytosolic NADPH hydrogen nuclei come from water via hydrogen-deuterium exchange. Such exchange does not account for any NADPH's high energy electrons, but 
merely dilutes deuterium tracer signal from the actual hydride donors like $[1-2 \mathrm{H}]$ glucose or $[1-2 \mathrm{H}]$ acetaldehyde (supplementary figure 6G,H,I,9). Correcting for such exchange (and for extent of substrate labeling), we observed that the oxPPP and Ald6 together account for most cytosolic NADPH, with the contribution of ethanol via Ald6 roughly double that of glucose via the oxidative pentose phosphate pathway (Figure 5D).

Consistent with ethanol oxidation and oxPPP being alternative cytosolic NADPH production pathways, in $\triangle$ ald6, oxPPP contribution to NADPH production (based on fatty acid labeling patterns) is nearly twice as high as in wild type (Figure 5C, supplementary figure 7). In $\Delta z w f 1$, Ald6 contribution to NADPH production (based on fatty acid labeling patterns) similarly doubles (Figure $5 \mathrm{~B}$, supplementary figure 8 ). Thus, ethanol is an important source of both acetyl and hydride units in Baker's yeast.

\section{Ethanol becomes a yet greater NADH and NADPH source in response to peroxide stress}

We were curious whether cells might shift between glucose or ethanol as an $\mathrm{NAD}(\mathrm{P}) \mathrm{H}$ source in response to environmental conditions. To explore this possibility, we grew yeast in glucose:ethanol with one substrate ${ }^{2} \mathrm{H}$ labeled as above, spiked in $\mathrm{H}_{2} \mathrm{O}_{2}$ to a final concentration of $20 \mathrm{mM}$, and rapidly sampled metabolites and their labeling ${ }^{18,19}$ (Figure 6A). Upon adding $\mathrm{H}_{2} \mathrm{O}_{2}$, the $\mathrm{NADH}$ concentration and $\mathrm{NADH} / \mathrm{NAD}^{+}$ratio fell markedly (Figure 6B, C). Such a drop was expected, given that oxidative stress is known oxidize the GADPH's active site cysteine and thereby block glycolytic flux and NADH production. Consistent with GAPDH being shut off, in addition to an increase of fructose-1,6-bisphosphate (FBP) (Supplementary Figure 10), we observed increased NADH labeling from ethanol, which became the dominant NADH hydride source (Figure 6D). Thus, ethanol catabolism is a crucial source for NADH when glycolysis is blocked by oxidative stress.

A classical rationale for glycolytic blockade by oxidative stress is to divert flux into the oxidative pentose phosphate pathway to help maintain NADPH homeostasis. Notably, the same concentration of hydrogen peroxide that markedly suppressed NADH had no overt effect on NADPH pool size or the NADPH: NADP+ ratio (Figure 6E, F). However, rather than increasing the oxidative pentose phosphate pathway contribution to $\mathrm{NADPH}$ as measured by $[1-2 \mathrm{H}]$ glucose, this contribution was decreased, with ethanol's contribution to NADPH markedly increased (Figure 6G). Thus, in contrast to the common assumption that the main NADPH production route during oxidative stress is the oxidative pentose phosphate pathway, we observe an acute shift towards a greater NADPH contribution from ethanol oxidation under $\mathrm{H}_{2} \mathrm{O}_{2}$ stress ${ }^{15,20}$.

\section{Discussion}

A fundamental metabolic question is, "Which pathways are coupled versus independent?" Here we present evidence that uncoupling of glycolysis from the TCA cycle is an evolutionarily conserved design principle in eukaryotic metabolism. Specifically, we show that fermenting Baker's yeast simultaneously release and uptake ethanol, much as many mammalian cells simultaneously produce and consume circulating lactate. Both lactate and ethanol are redox-balanced with glucose ${ }^{21}$. Thus, their release allows glycolysis to run without need for the TCA cycle or oxidative phosphorylation. Release of these electron-rich products anticorrelates with internal $\mathrm{NADH}$ consumption by the electron transport chain 22 . 
While the net release of ethanol by fermenting yeast has been long appreciated, we are unaware of prior demonstration that fermenting yeast simultaneously engage in extensive ethanol uptake. Through experiments with ${ }^{13} \mathrm{C}$-ethanol, we show that, under typical mid-exponential fermentative growth conditions, environmental ethanol, rather than mitochondrial pyruvate catabolism, supplies a majority of both cytoso lic and mitochondrial acetyl-CoA. In mitochondria, we prove that the ethanol assimilation pathway involves the CoA-transferase Ach123-25. The assimilated ethanol was originally produced from glucose. But the pathway from glycolysis to the TCA cycle (and other acetyl-CoA products like fatty acids and amino acids) flows through pyruvate decarboxylase and environmental ethanol.

This metabolic design simplifies regulation of the fate of pyruvate, circumventing the challenge of partitioning pyruvate optimally between ethanol and acetyl-CoA. Ethanol excretion is the default, and required two-carbon units are re-assimilated as needed from environmental ethanol. This design further assures adequate availability of both carbon and high-energy electrons even if glycolysis is impaired. Without any remodeling of their internal metabolic machinery, yeast have access to both carbon and high-energy electrons from environmental ethanol. Such access is particularly evident during acute redox stress, which impairs glycolysis through inhibitory oxidation of the central glycolytic enzyme GAPDH. Under this circumstance, ethanol becomes the predominant source of both $\mathrm{NADH}$ and $\mathrm{NADPH}$, the latter being critical for survival of oxidative stressors. Thus, uncoupling of glycolysis from the TCA cycle via ethanol provides yeast with metabolic flexibility, decreases regulatory complexity, and enhances robustness.

\section{Methods}

Materials. Yeast were grown in yeast nitrogen base without amino acids (Sigma Aldrich, Y0626) with carbon source added separately. BD Difco TM YPD Broth (BD 242820) was used as the media for reviving frozen cells or growing cells to be frozen. Glycerol (Sigma Aldrich, G5516) was added at 1:1 volume ratio to YPD yeast cultures in cryovials (Nalgene $\AA$, 5000-1020, or Corning, 430289). Glucose (Sigma Aldrich, D9434), [U${ }^{13} \mathrm{C}_{6}$ ]glucose (Cambridge Isotope Laboratories, CLM-1396), $\left[{ }^{13} \mathrm{C}_{2}\right]$ ethanol (Sigma Aldrich, 427039), [1,1${ }^{2} \mathrm{H}_{2}$ ] ethanol (Sigma Aldrich, 347434), ethanol (Decon Labs Inc., DSP-MD.43), and 1-2H-glucose (Omicron Biochemistry, GLC-032) are used as carbon sources. Tap water filtered by Milli-Q® Reference Water Purification System (Millipore Sigma, C79625) and $\mathrm{D}_{2} \mathrm{O}$ (Cambridge Isotope Laboratories, DLM-4) were used as the water source for yeast cultures. MilliporeSigma ${ }^{\mathrm{TM}}$ Stericup ${ }^{\mathrm{TM}}$ Quick Release-HV Sterile Vacuum Bottle Top Filtration Systems with 0.22 um PES filters were used to sterilize all media for yeast cultures. $0.45 \mu \mathrm{m}$ nylon filters (GVS North America, 123776) were used to filtering yeasts for metabolite extraction. 3(Trimethylsilyl)propionic-2,2,3,3-d4 acid sodium salt (TMSP) (Sigma Aldrich, 269913) was used as ${ }^{1} \mathrm{H} N M R$ internal standard and sodium formate- ${ }^{13} \mathrm{C}$ (Sigma Aldrich, 279412) was used ${ }^{13} \mathrm{C}$ NMR internal standard. Experimental cultures were grown in $14 \mathrm{~mL}$ polypropylene round bottom tubes (FALCON, 352059). HPLC vials (Thermo Scientific, 200-046, 501-313) were used for water-soluble metabolite samples and glass vials (Fisher Scientific, 03-338A) were used for lipid hydrolysis and glass HPLC vials (Chemglass, CV-1152-1232, CV-384513009) were used for fatty acid and lipid samples. 
Yeast strains. S. cerevisiae strain FY4, derived from S288c, was taken from in-house frozen stocks. S. cerevisiae strain CEN.PK was obtained from José Avalos. S. cerevisiae prototrophic mutant strains were obtained from David Botstein, which were also derived from S288c through a diploid intermediate strain ${ }^{26,27}$.

Yeast batch cell culture growth. S. cerevisiae colony was inoculated into an overnight culture, containing 6.7 $\mathrm{g} / \mathrm{L}$ of Yeast Nitrogen Base (YNB) without amino acids and $20 \mathrm{~g} / \mathrm{L}$ of glucose. After $24 \mathrm{~h}$ of growth at $30^{\circ} \mathrm{C}$, the overnight culture was diluted 1:100 into appropriate experimental media, containing $6.7 \mathrm{~g} / \mathrm{L}$ YNB without amino acids and a carbon source/isotope tracer as specified for each experiment. For the prototrophic $\Delta z w f 1$ strain, $20 \mathrm{mg} / \mathrm{L}$ methionine was added to the media to accelerate growth.

${ }^{1} \mathrm{H}$ qNMR measurement. Yeast were first grown to mid-exponential phase (OD=0.5), split into two equal portions, and centrifuged to pellets. In the equimolar case, each pellet was redissolved at equal concentration into media containing either (i) YNB + $42 \mathrm{mM}$ unlabeled glucose and $42 \mathrm{mM}$ [U-13C]ethanol or (ii) YNB+ $42 \mathrm{mM}$ $\left[U-{ }^{13} \mathrm{C}\right]$ glucose and $42 \mathrm{mM}$ unlabeled ethanol. In the equicarbon case, each portion was switched into the media containing either (i) YNB +28 mM unlabeled glucose and $84 \mathrm{mM}\left[\mathrm{U}-{ }^{12} \mathrm{C}\right]$ ethanol or (ii) YNB+ $28 \mathrm{mM}[\mathrm{U}-$ ${ }^{13} \mathrm{C}$ ]glucose and $84 \mathrm{mM}$ unlabeled ethanol. OD was taken before and after the incubation period for flux calculation. Before switching and 1 hour after the media switch, $0.5 \mathrm{~mL}$ of the culture media was collected by sampling and centrifugation. $450 \mu \mathrm{L}$ of the supernatant was mixed with $50 \mu \mathrm{L}$ of the $50 \mathrm{mM}$ deuterated TMSP standard solution in $\mathrm{D}_{2} \mathrm{O}$ and loaded onto NMR machine. $1 \mathrm{H}$ NMR $\delta: 0$ ppm (s, 9H) for the TMS standard, 3.2 ppm (dd, $1 \mathrm{H})$ for $\beta$-glucose, and $1.17 \mathrm{ppm}(\mathrm{t}, 3 \mathrm{H})$ for ethanol. Water suppression is achieved by $\mathrm{O}_{1} \mathrm{P}=4.66$ ppm, spw1 $1=0.002$. To achieve quantitative NMR, $D_{1}$ is set to $5 \mathrm{~s}$ and $90^{\circ}$ pulse ( $p_{1}$ )to $11.69 \mu \mathrm{s}$.

${ }^{13} \mathrm{C}$ qNMR measurement. The sample collection procedure and preparation were identical to the ${ }^{1} \mathrm{H}$ NMR excretion profile, except that ${ }^{13} \mathrm{C}$ sodium formate $(0.1 \mathrm{M})$ was utilized as a standard instead of deuterated TMS.

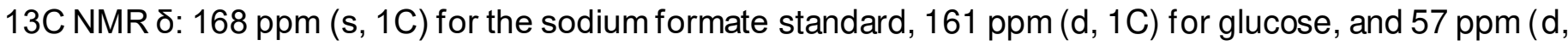
1C) for ethanol. Samples of media that the cells are switched into are measured as baseline. We observed that unlabeled ethanol present in the media also gives a detectable ${ }^{13} \mathrm{C}$ NMR signal (s, $1 \mathrm{C}$ at $57 \mathrm{ppm}$ ) due to natural ${ }^{13} \mathrm{C}$ abundance. This natural abundance signal was not corrected for because it is $<5 \%$ signal of [U${ }^{13} \mathrm{C}$ ]ethanol. Relaxation time ( $\left.D_{1}\right)$ is set to a generous length of $40 \mathrm{~s}$ to ensure all spins reset properly and thus achieve quantification of ${ }^{13} \mathrm{C}$ nuclei.

Metabolite extraction. Metabolite extraction was performed according as previously $28,29.3 \mathrm{~mL}$ of the cell culture at mid-exponential stage of growth $(O D \sim 0.6)$ was vacuum filtered $(0.45 \mu \mathrm{m}$, Millipore). The filter was immersed in $1.6 \mathrm{~mL}$ of a cold $\left(-20^{\circ} \mathrm{C}\right)$ extraction buffer (40\% methanol: $40 \%$ acetonitrile: $20 \%$ water: $0.5 \%$ formic acid by volume). Cells were washed out of the filter, and $88 \mu \mathrm{L}$ of $\mathrm{NH}_{4}\left(\mathrm{HCO}_{3}\right)(15 \% \mathrm{w} / \mathrm{v}$, Sigma-Aldrich) solution per $1 \mathrm{~mL}$ extraction buffer was added to neutralize formic acid. The resulting solutions were transferred to Eppendorf tubes and centrifuged for 10 min at 16,000 rotations per minute (rpm) in a cold room $\left(4^{\circ} \mathrm{C}\right)$. The supernatant was collected and stored at $-80^{\circ} \mathrm{C}$ before loading onto a liquid chromatography-mass spectrometer (LC-MS). 
Water-soluble metabolite LC-MS Analysis. Prepared samples were loaded onto a quadrupole-orbitrap mass spectrometer (Q Exactive Plus, Thermo Fisher Scientific) coupled to hydrophilic interaction chromatography (HILIC) for analysis. Measurements of acetyl-CoA labeling were achieved by reversed-phase ion-pairing liquid chromotography ${ }^{30}$ coupled to a standalone orbitrap (Exactive, Thermo Fischer Scientific).

Fatty acid extraction and LC-MS analysis. Fatty extraction was performed according to Zhang et al ${ }^{16}$. Cells were pelleted in a $1.5 \mathrm{~mL}$ Eppendorf tube, and $1 \mathrm{~mL}$ of $0.3 \mathrm{M} \mathrm{KOH}$ in 90:10 methanol: water solution was added. The resulting mixture with cells was transferred to a $4 \mathrm{~mL}$ glass vial. Saponification was performed by placing the samples into a water bath at $80^{\circ} \mathrm{C}$ for one hour. Once the samples cooled down, $100 \mu \mathrm{L}$ formic acid $(0.5 \%)$ was added, followed by $1 \mathrm{~mL}$ hexane to extract the fatty acids. The extract was transferred into a glass HPLC vial and dried under nitrogen flow. Afterward, it was diluted in 0.1 mL 50:50 acetonitrile: methanol solution. The $0.1 \mathrm{~mL}$ solution was then added to a clean glass insert and placed inside an HPLC vial and the cap sealed. All the samples were loaded onto the Exactive LC-MS employing a reversed-phase LC column (C8) coupled with negative-mode ESI high-resolution MS. NADPH or acetyl-CoA labeled fractions were inferred from observed fatty acid labeling patterns using a binomial model With unlabeled fat, which in part reflects environmental contamination, omitted from the calculation.

Isotope tracing experiments. Cells were grown in YNB $+10 \mathrm{~g} / \mathrm{L}$ glucose up to $\mathrm{OD}=0.5$. Then, the cells were quickly centrifuged, the supernatant discarded, and switched to equimolar or equicarbon media with either glucose or ethanol ${ }^{13} \mathrm{C}$ - or ${ }^{2} \mathrm{H}$-labeled, or with both carbon sources unlabeled in $50 \%{ }^{2} \mathrm{H}_{2} \mathrm{O}$. The cells were allowed to grow in the labeled media for 1 hour before harvesting. A potential concern these experiments is dilution of labeled ethanol tracer by unlabeled ethanol made from glucose. As no more than $2 \mathrm{mM}$ unlabeled ethanol is excreted into the media during the one-hour incubation, environmental ethanol remains more than $95 \%$ fully labeled during the experiments. OD was taken before and after the incubation period to assure the cells were in exponential growth phase during the experiment.

Substrate correction for deuterium tracing with [1,1-2 $\left.\mathrm{H}_{2}\right]$ ethanol. PDC flux dilutes the deuterium labeled fraction of acetaldehyde in S. cerevisiae when fed with $\left[1,1-2 \mathrm{H}_{2}\right]$ ethanol. Contribution to NADPH by Ald6 is represented by the labeled NADPH active hydride fraction from $\left[1,1-2 \mathrm{H}_{2}\right]$ ethanol divided by acetaldehyde labeling. The acetaldehyde labeling is approximated by the fraction of acetyl-CoA labeled by ethanol in ${ }^{13} \mathrm{C}$ isotope tracing experiments. Due to the deuterium kinetic isotope effect, and PDH flux being small, if anything this will overestimate ${ }^{2} \mathrm{H}$-labeling of acetaldehyde, and thus tend to underestimate Ald6 contribution to NADPH. Was

Water exchange activity calculation with ${ }^{2} \mathrm{H}_{2} \mathrm{O}$ experiments. The fraction of NADPH in exchange with water is represented by the fraction of NADPH active hydride labeled from ${ }^{2} \mathrm{H}_{2} \mathrm{O}$ divided by the ${ }^{2} \mathrm{H}_{2} \mathrm{O}$ enrichment percentage (50\%).

$\mathrm{H}_{2} \mathrm{O}_{2}$ stress experiments. The procedure was adapted from Christodoulou et al. with some modifications ${ }^{19}$. Cells were incubated in the experimental culture media, containing either $\left[1,1-{ }^{2} \mathrm{H}_{2}\right]$ ethanol or $\left[1-{ }^{2} \mathrm{H}\right] \mathrm{glucose}$ tracer, during the exponential growth stage. Metabolite extraction was performed with $1 \mathrm{~mL}$ of the culture for a 
baseline measurement ("- $1 \mathrm{~s}$ " time point). Afterward, 6 uL of $30 \%$ hydrogen peroxide (Sigma Aldrich) was added to the $3 \mathrm{~mL}$ culture to reach a final concentration of $20 \mathrm{mM} \mathrm{H}_{2} \mathrm{O}_{2}$ and samples were extracted after 15 , 30 , and $60 \mathrm{~s}$.

Mitochondrial acetyl-CoA labeling calculation from $\left[\mathrm{U}^{-{ }^{13}} \mathrm{C}_{2}\right]$ ethanol tracer. Acetyl-glutamate and $\alpha-$ ketoglutarate labeling patterns were measured from the LC-MS analysis of cultures grown with $\left[\mathrm{U}^{1{ }^{13}} \mathrm{C}_{2}\right]$ ethanol. The matrix equation was set up as below, and solved by the least square method for the acetyl-CoA labeling.

$$
\left[\begin{array}{ccc}
M+0 & 0 & 0 \\
M+1 & M+0 & 0 \\
M+2 & M+1 & M+0 \\
0 & M+2 & M+1 \\
0 & 0 & M+2
\end{array}\right]\left(\begin{array}{l}
M+0 \\
M+1 \\
M+2
\end{array}\right)=\left(\begin{array}{l}
M+0 \\
M+1 \\
M+2 \\
M+3 \\
M+4
\end{array}\right)
$$

a-ketoglutarate acetyl-CoA acetyl-glutamate

Data analysis and visualization. EI-MAVEN v11.1 (Elucidata) software was used to process the LC-MS data ${ }^{31}$. Metabolite identities were verified by both mass/charge $(\mathrm{m} / \mathrm{z})$ ratio and retention time match to authenticated standards. For ${ }^{2} \mathrm{H}$ - and ${ }^{13} \mathrm{C}$-isotope-labeled data analysis, natural isotope abundance correction was made according to the binomial distribution model ${ }^{32} .{ }^{13} \mathrm{C}$-MFA was computed with INCA ${ }^{33}$. NADPH active hydride labeling and acetyl-CoA labeling from fatty acids were calculated as previously ${ }^{16}$. MestReNova 64 software was used for the NMR data processing. Statistical analysis are performed with GraphPad Prism, including twotailed t-test (with false discovery rate correction by the two-stage step up Benjamini, Krieger, and Yekutieli method to confirm that any reported significant results involving statistical comparisons of multiple isotopic forms of the same metabolite remain significant after correction for the multiple comparisons); ordinary oneway ANOVA (when row matching is statistically significant, RM one-way ANOVA instead); and linear trend (between column mean and by left-to-right column order, with $\mathrm{p}$-value from $\mathrm{F}$ test). For routine data visualization and analysis, MATLAB ${ }^{34}, \mathrm{R}$ Studio, Python, and Microsoft Excel were utilized. Schematics and diagrams were created with the aid of GraphPad Prism, ChemDraw, and BioRender.

\section{References}

1. Weinhouse, S., Warburg, O., Burk, D. \& Schade, A. L. On respiratory impairment in cancer cells. Science (80-. ). 124, 267-272 (1956).

2. Wolfe, A. J. The Acetate Switch. Microbiol. Mol. Biol. Rev. 69, 12-50 (2005).

3. De Deken, R. H. The Crabtree effect: a regulatory system in yeast. J. Gen. Microbiol. 44, 149-156 (1966).

4. Bui, L. T. et al. Conservation of ethanol fermentation and its regulation in land plants. J. Exp. Bot. 70, 1815-1827 (2019).

5. Hui, S. et al. Glucose feeds the TCA cycle via circulating lactate. Nature 551, 115-118 (2017).

6. Arthur L. Kruckeberg and J. Richard Dickinson. Metabolism and Molecular Physiology of Saccharomyces Cerevisiae. Metabolism and Molecular Physiology of Saccharomyces Cerevisiae 
(Taylor \& Francis Ltd, 2004). doi:10.1201/9780203503867

7. Verduyn, C., Zomerdijk, T. P. L., van Dijken, J. P. \& Scheffers, W. A. Continuous measurement of ethanol production by aerobic yeast suspensions with an enzyme electrode. Appl. Microbiol. Biotechnol. $198419319,181-185$ (1984).

8. DeRisi, J. L., lyer, V. R. \& Brown, P. O. Exploring the metabolic and genetic control of gene expression on a genomic scale. Science (80-. ). 278, 680-686(1997).

9. Kotyk, A. \& Alonso, A. Transport of ethanol in baker's yeast. Folia Microbiol. (Praha). 30, 90-91 (1985).

10. Pronk, J. T., Yde Steensma, H. \& Van Dijken, J. P. Pyruvate metabolism in Saccharomyces cerevisiae. Yeast 12, 1607-1633 (1996).

11. Van den Berg, M. A. et al. The two acetyl-coenzyme A synthetases of Saccharomyces cerevisiae differ with respect to kinetic properties and transcriptional regulation. J. Biol. Chem. 271, 28953-28959 (1996).

12. Jong-Gubbels, P., Berg, M. A., Steensma, H. Y., Dijken, J. P. \& Pronk, J. T. The Saccharomyces cerevisiae acetyl-coenzyme A synthetase encoded by the ACS1 gene, but not the ACS2-encoded enzyme, is subject to glucose catabolite inactivation. FEMS Microbiol. Lett. 153, 75-81 (2006).

13. Buu, L. M., Chen, Y. C. \& Lee, F. J. S. Functional characterization and localization of acetyl-CoA hydrolase, Ach1p, in Saccharomyces cerevisiae. J. Biol. Chem. 278, 17203-17209 (2003).

14. Otterstedt, K. et al. Switching the mode of metabolism in the yeast Saccharomyces cerevisiae. EMBO Rep. 5, 532-537 (2004).

15. Grabowska, D. \& Chelstowska, A. The ALD6 gene product is indispensable for providing NADPH in yeast cells lacking glucose-6-phosphate dehydrogenase activity. J. Biol. Chem. 278, 13984-13988 (2003).

16. Zhang, Z., Chen, L., Liu, L., Su, X. \& Rabinowitz, J. D. Chemical Basis for Deuterium Labeling of Fat and NADPH. J. Am. Chem. Soc. 139, 14368-14371 (2017).

17. TE, B., JL, M., AC, M., FA, E. \& CT, W. Overexpression, purification, and mechanistic study of UDP-Nacetylenolpyruvylglucosamine reductase. Biochemistry 32, 2024-2030 (1993).

18. Collinson, L. P. \& Dawes, I. W. Inducibility of the response of yeast cells to peroxide stress. J. Gen. Microbiol. 138, 329-335 (1992).

19. Christodoulou, D. et al. Reserve Flux Capacity in the Pentose Phosphate Pathway by NADPH Binding Is Conserved across Kingdoms. iScience 19, 1133-1144 (2019).

20. Minard, K. I. \& Mcalister-henn, L. Sources of NADPH in Yeast Vary with Carbon Source *. J. Biol. Chem. 280, 39890-39896 (2005).

21. van Hoek, M. J. A. \& Merks, R. M. H. Redox balance is key to explaining full vs. partial switching to low yield metabolism. BMC Syst. Biol. 6, 22 (2012).

22. Vemuri, G. N., Eiteman, M. A., McEwen, J. E., Olsson, L. \& Nielsen, J. Increasing NADH oxidation reduces overflow metabolism in Saccharomyces cerevisiae. Proc. Natl. Acad. Sci. U. S. A. 104, 24022407 (2007).

23. Lee, F. J. S., Lin, L. W. \& Smith, J. A. Acetyl-CoA hydrolase involved in acetate utilization in Saccharomyces cerevisiae. Biochim. Biophys. Acta - Protein Struct. Mol. Enzymol. (1996). doi:10.1016/0167-4838(96)00109-4

24. Fleck, C. B. \& Brock, M. Re-characterisation of Saccharomyces cerevisiae Ach1p: Fungal CoAtransferases are involved in acetic acid detoxification. Fungal Genet. Biol. 46, 473-485 (2009).

25. Chen, Y., Zhang, Y., Siewers, V. \& Nielsen, J. Ach1 is involved in shuttling mitochondrial acetyl units for cytosolic C2 provision in Saccharomyces cerevisiae lacking pyruvate decarboxylase. FEMS Yeast Res. 
15, 1-8 (2015).

26. Gibney, P. A., Lu, C., Caudy, A. A., Hess, D. C. \& Botstein, D. Yeast metabolic and signaling genes are required for heat-shock survival and have little overlap with the heat-induced genes. Proc. Natl. Acad. Sci. U. S. A. 110, E4393-402 (2013).

27. VanderSluis, B. et al. Broad metabolic sensitivity profiling of a prototrophic yeast deletion collection. Genome Biol. 15, R64 (2014).

28. Bennett, B. D., Yuan, J., Kimball, E. H. \& Rabinowitz, J. D. Absolute quantitation of intracellular metabolite concentrations by an isotope ratio-based approach. Nat. Protoc. 3, 1299-1311 (2008).

29. Park, J. O. et al. Metabolite concentrations, fluxes and free energies imply efficient enzyme usage. Nat. Chem. Biol. 12, 482-489 (2016).

30. Lu, W. et al. Metabolomic Analysis via Reversed-Phase Ion-Pairing Liquid Chromatography Coupled to a Stand Alone Orbitrap Mass Spectrometer. Anal. Chem. 82, 3212-3221 (2010).

31. Agrawal, S. et al. EL-MAVEN: A fast, robust, and user-friendly mass spectrometry data processing engine for metabolomics. in Methods in Molecular Biology 1978, 301-321 (Humana Press Inc., 2019).

32. Su, X., Lu, W. \& Rabinowitz, J. D. Metabolite Spectral Accuracy on Orbitraps. Anal. Chem. 89, 5940$5948(2017)$.

33. Young, J. D. INCA: a computational platform for isotopically non-stationary metabolic flux analysis. Bioinformatics 30, 1333-1335 (2014).

34. MATLAB. 9.7.0.1190202 (R2019b). (The MathWorks Inc., 2018).

\section{Acknowledgements}

We thank S. Silverman and D. Botstein for access to the yeast knockout collection, T.TeSlaa, R. Ryseck, and A.J. Cowan for feedback on the manuscript, and I. Pelczer and J. Eng for assistance with NMR and mass spectrometry, C.M. Call for help with preliminary experiments, and M. Seyedsayamdost and members of the Rabinowitz lab for helpful discussions. Services, results and/or products in support of the research project were generated by the Rutgers Cancer Institute of New Jersey Metabolomics Shared Resource, supported, in part, with funding from NCI-CCSG P30CA072720-5923. This work was supported by DOE grant DE SC0018420 (CABBI).

\section{Author contributions}

T.X. and J.D.R. designed the study. T.X. performed NMR studies. T.X. and A.K. performed isotopic tracing studies. T.X., Y.H., L.C. and A.K. developed the computational models. T.X. and A.K. analyzed the data. J.D.R. and T.X. wrote the manuscript with the help from all authors.

\section{Competing interests}

None.

\section{Materials \& Correspondence}

Correspondence and requests for materials should be addressed to J.D.R.

\section{Additional Information}

Supplementary Information is available for this paper. 


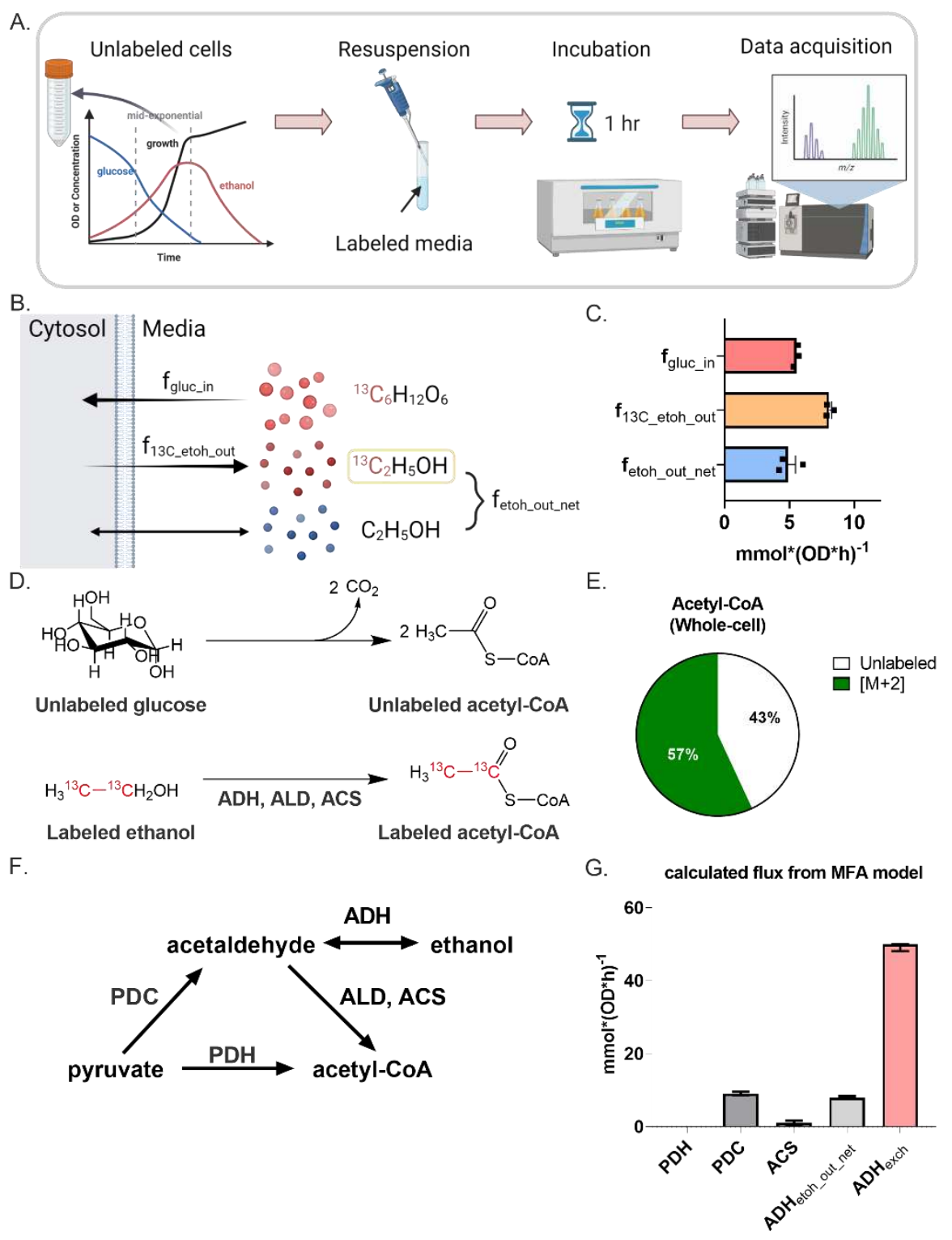

Figure 1. Environmental ethanol is a major source of acetyl units in fermenting S. cerevisiae.

A. Schematic of the experimental design, in which fermenting yeast are transferred into fresh media containing both glucose and ethanol, whose isotopic composition is under experimenter control (Created with Biorender).

B. Approach to measuring uptake/excretion fluxes. S. cerevisiae is switched into ${ }^{13} \mathrm{C}$-glucose + unlabeled ethanol media, and net glucose consumption (fgluc in) and total production of ethanol from glucose ( $f_{13 C}$ _etoh_out) are measured by ${ }^{13} \mathrm{C}-\mathrm{NMR}$. The net ethanol flux (of combined labeled and unlabeled ethanol) is measured by ${ }^{1} \mathrm{H}-\mathrm{NMR}$ in a parallel experiment based on concentration change of the total ethanol pool (fetoh_out_net).

C. Uptake/excretion fluxes for S. cerevisiaeFY4 in minimal media (YNB) with equimolar [U- $\left.{ }^{13} \mathrm{C}\right] g l u c o s e:$ unlabeled ethanol (mean, SE, $n=3$ (biological replicates)).

D. When $S$. cerevisiae is switched into unlabeled glucose $+{ }^{13} \mathrm{C}$-ethanol media, ethanol oxidation results in $[\mathrm{M}+2]$ acetyl-CoA, which is detectable by LC-MS.

E. The whole-cell [M+2] acetyl-CoA fraction (mixture of cytosolic and mitochondrial origins) for $S$. cerevisiae FY4 in minimal media (YNB) with equimolar glucose: $\left[{ }^{13} \mathrm{C}_{2}\right]$ ethanol (mean, $\mathrm{n}=3$ (biological replicates)).

F. Metabolic fluxes relevant to pyruvate and ethanol metabolism in yeast.

G. ${ }^{13} \mathrm{C}$-metabolic flux analysis based on model in $\mathbf{F}$ and data in $\mathbf{C}$ and $\mathbf{E}$. PDC flux is substantial while PDH flux is below detection, signifying acetaldehyde is the direct contributor for acetyl-CoA. High exchange flux at ADH implicates environmental ethanol as a major contributor to cellular acetaldehyde and acetyl$\mathrm{CoA}$ (mean, UB/LB, $\mathrm{n}=3$ (biological replicates)). 
A.

<smiles>CCC[13C](=O)SC(=O)OCCCCCCCC(=O)SC(C)=O</smiles>

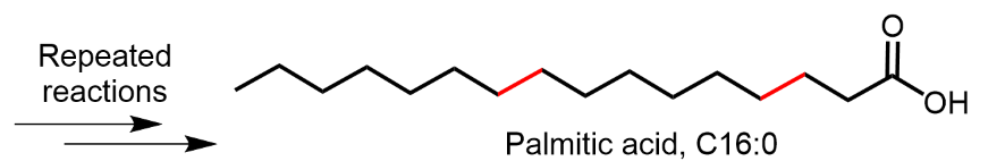

B. Fatty acid labeling (C16:0)

C.<smiles>CCCCCCCCCCCCCCCCCC(=O)O</smiles>
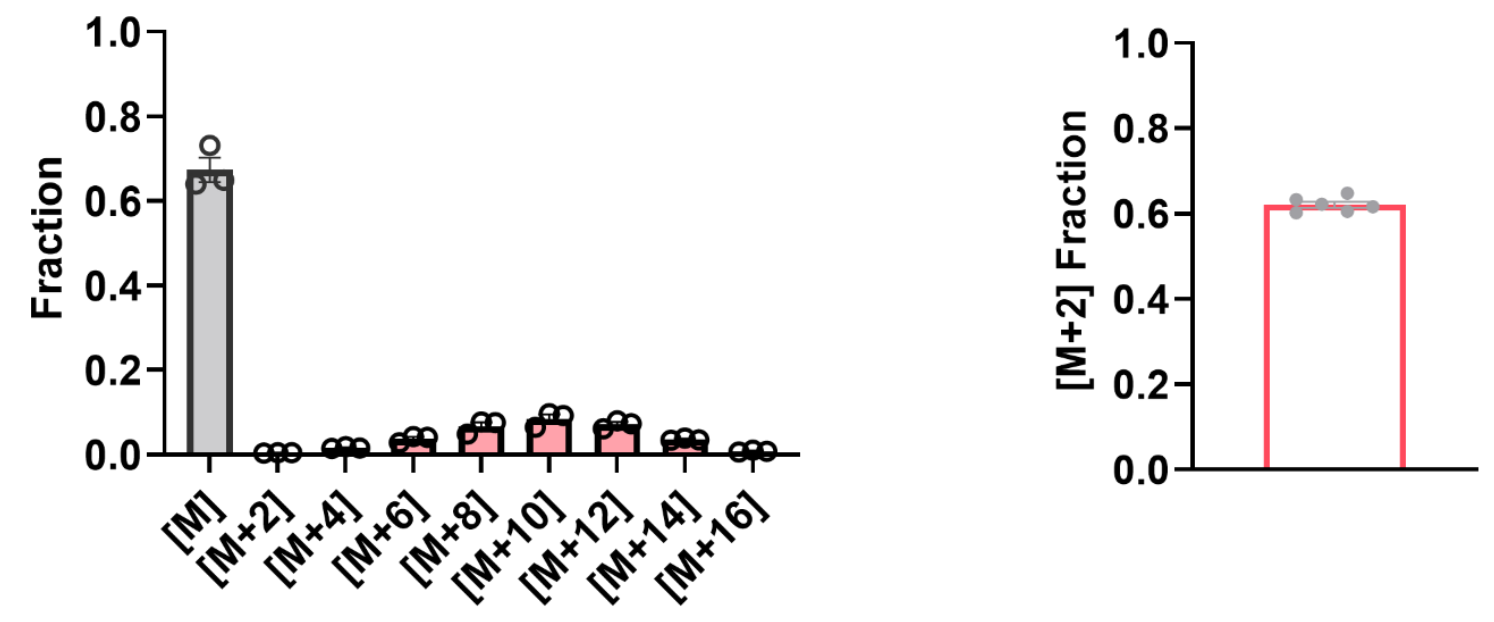

Figure 2. Environmental ethanol feeds fatty acid synthesis in fermenting S. cerevisiae.

A. When fed with labeled ethanol, the resulting ${ }^{13} \mathrm{C}$-labeled acetyl-CoA is incorporated into newly synthesized fatty acids. As both labeled and unlabeled cytosolic acetyl-CoA are randomly incorporated into growing fatty acid chains, the resulting fatty acid mass isotope distribution follows a binomial probability distribution.

B. Fatty acid (palmitate) labeling pattern from equimolar glucose: ${ }^{13} \mathrm{C}$-ethanol co-feeding experiment as in Figure 1D (mean, SE, $n=3$ (biological replicates)). Briefly, newly synthesized fatty acids are getting labeled by $[\mathrm{M}+2]$ acetyl-CoA, which is a result of uptaking ${ }^{13} \mathrm{C}$-ethanol from growth media by $S$. cerevisiae.

C. Cytosolic acetyl-CoA labeling fitted from fatty acid labeling from equimolar glucose: ${ }^{13} \mathrm{C}$-ethanol cofeeding experiment as in Figure 1D (mean, SE, $n=6$ (3 biological replicates with results from both C16:0 and C18:0)). 


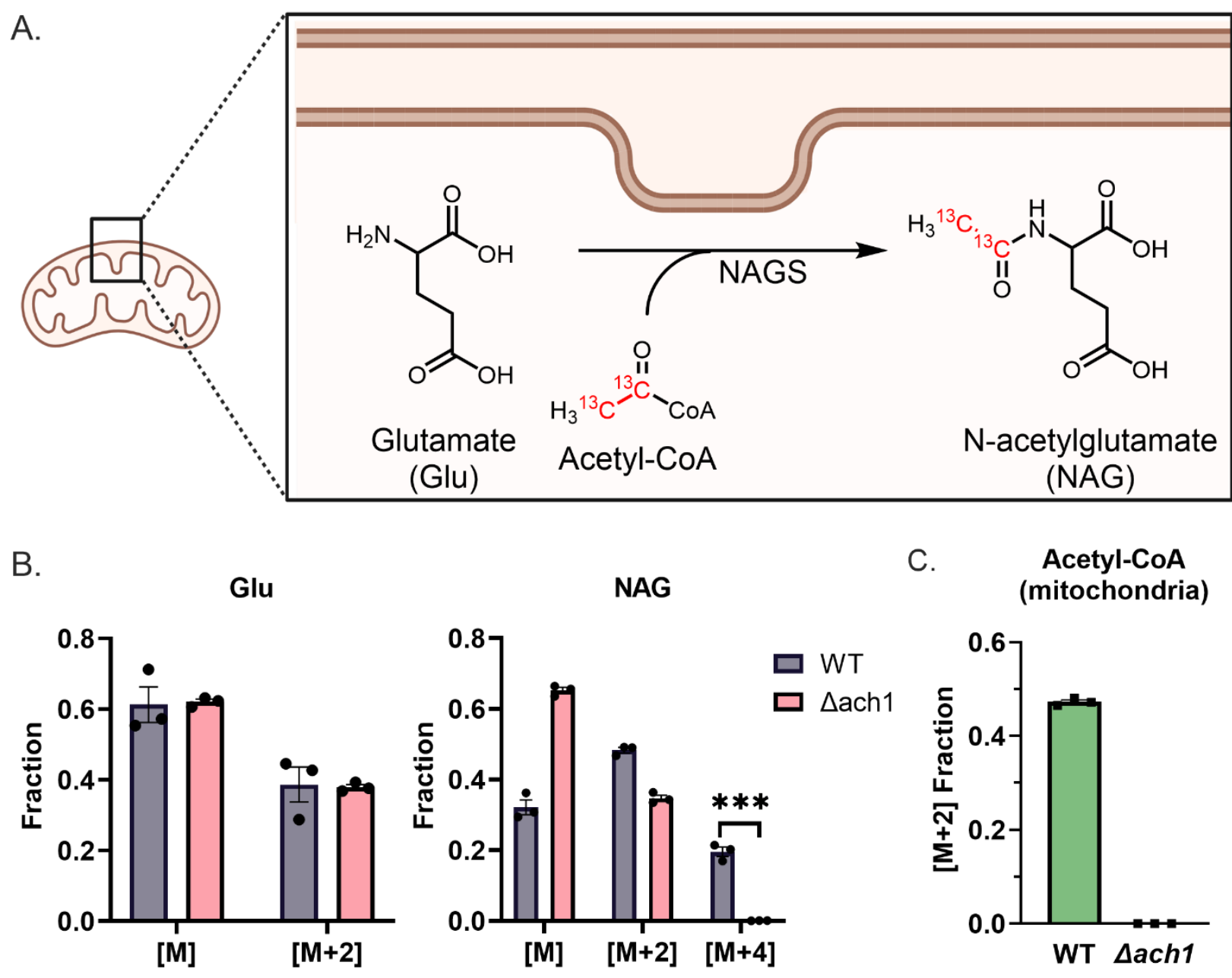

Figure 3. Environmental ethanol contributes to amino acid synthesis through mitochondrial acetylCoA.

A. Synthesis of the arginine precursor N-acetylglutamate (NAG) in S. cerevisae takes place in mitochondria (Created with Biorender). A linear algebra deconvolution of the labeling fractions of glutamate and NAG can compute the mitochondrial acetyl-CoA labeling.

B. Glutamate (Glu) and NAG labeling from the glucose: ${ }^{13} \mathrm{C}$-ethanol co-feeding experiment as in Figure 1D, including also data for $\Delta$ ach 1 yeast (thereby identifying Ach1 as an enzyme essential for mitochondrial assimilation of ethanol-derived carbon into acetyl-CoA) (mean, SE, $n=3$ (biological replicates); ${ }^{* * *}, \mathrm{p}<.001$ by t-test). Briefly, given the observed highest isotopic label of Glu is [M+2], if mitochondrial acetyl-CoA are labeled $[\mathrm{M}+2]$ by ${ }^{13} \mathrm{C}$-ethanol uptaken by $\mathrm{S}$. cerevisiae from growth media, newly synthesized NAG will have $[\mathrm{M}+4]$ isotopic label.

C. Mitochondrial acetyl-CoA [M+2] fraction fitted from glutamate and NAG labeling in $\mathbf{B}$ (mean, SE, $n=3$ (biological replicates)). 
A.



B.
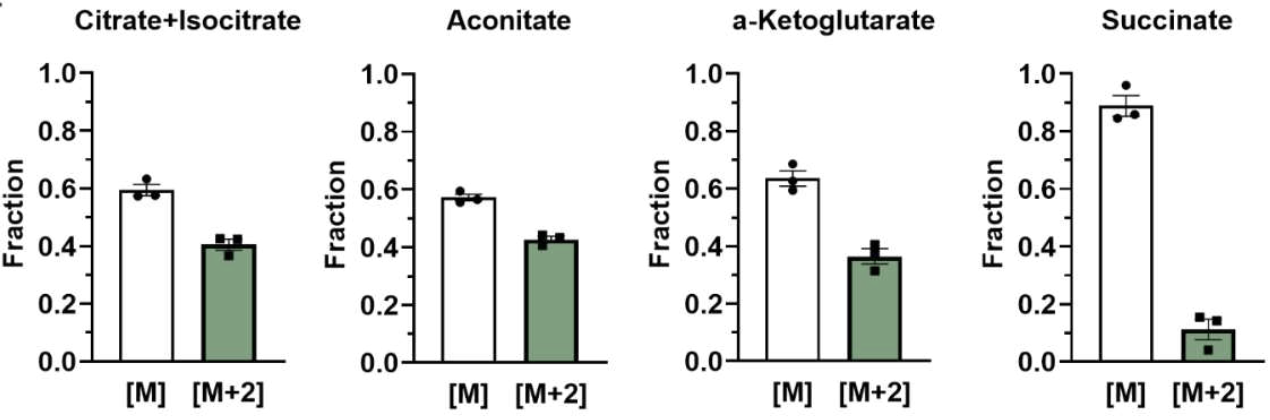

C.

Pyruvate
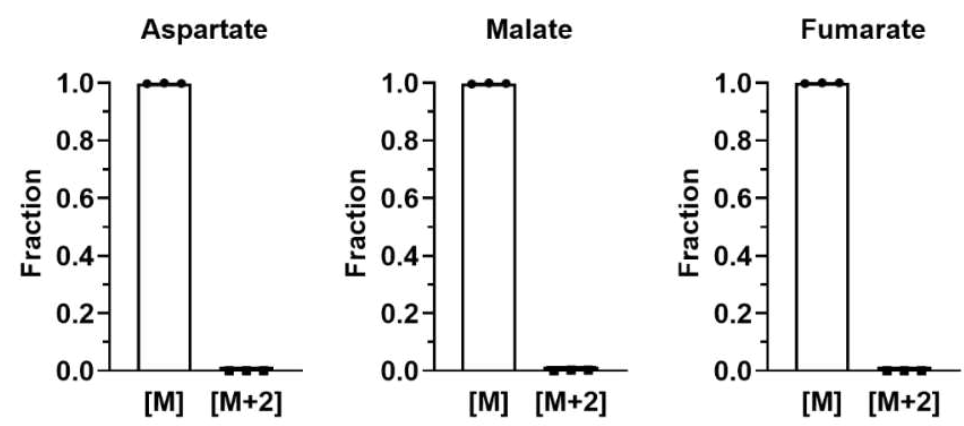

Figure 4. Carbons from ethanol feed into TCA intermediates.

A. Schematic of TCA cycle highlighting observed TCA labeling from glucose: ${ }^{13} \mathrm{C}$-ethanol co-feeding as in Figure 1D, where [M+2] acetyl-CoA is a result of uptaking ${ }^{13} \mathrm{C}$-ethanol from growth media by $S$. cerevisiae (Created with Biorender). ${ }^{13} \mathrm{C}$ is in green while ${ }^{12} \mathrm{C}$ is in white.

B. Labeling patterns of TCA intermediates on "right hand side" of TCA cycle (i.e. between citrate and succinate) (mean, SE, $n=3$ (biological replicates)).

C. Labeling patterns of pyruvate and TCA intermediates on "left hand side" of TCA cycle (with aspartate as a surrogate for oxaloacetate) (mean, $\mathrm{SE}, \mathrm{n}=3$ (biological replicates)). These metabolites are not labeled and thus originate exclusively from glucose, suggesting blockade of complex II and production of malate/fumarate through oxaloacetate reduction. 
A.

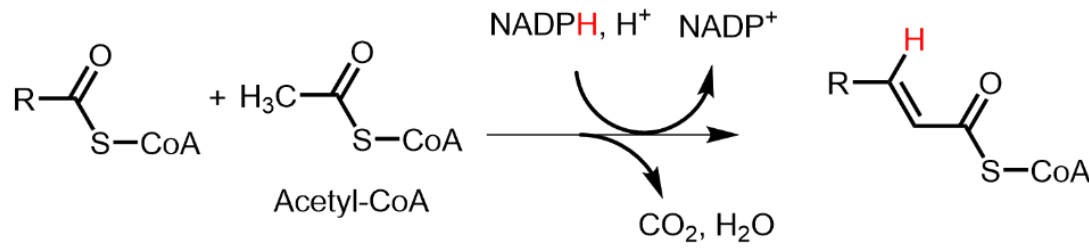

$\mathrm{NADPH}, \mathrm{H}^{+} \mathrm{NADP}^{+}$
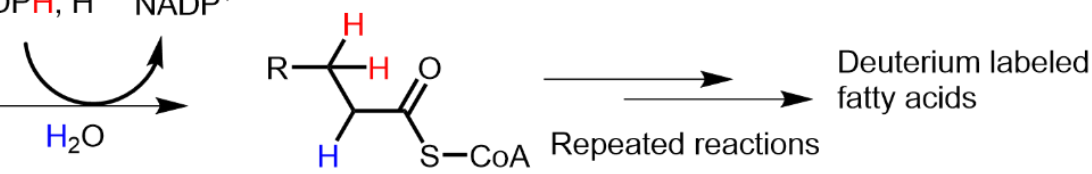

B.

Ald to NADPH (labeled ethanol)

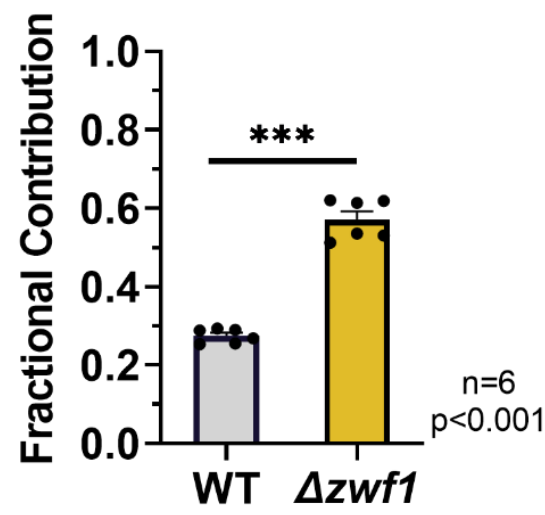

C.

$$
\begin{aligned}
& \text { oxPPP to NADPH } \\
& \text { (labeled glucose) }
\end{aligned}
$$

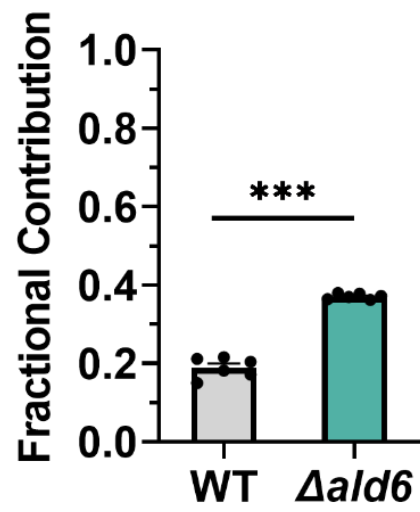

D.

\section{Contribution to cytosolic NADPH}

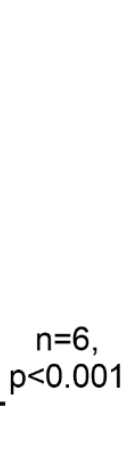

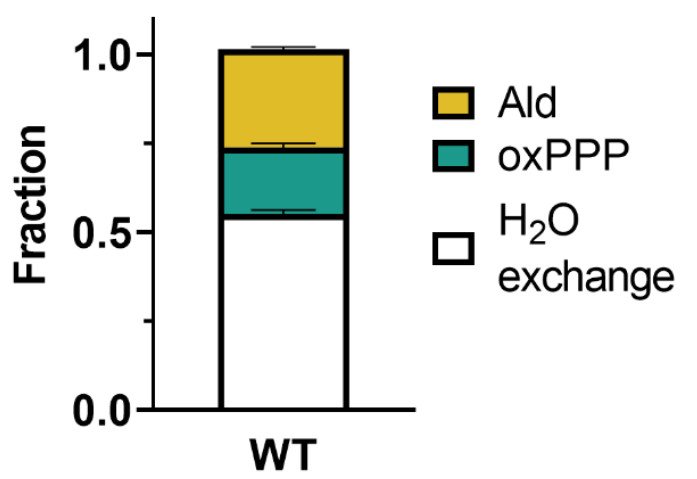

Figure 5. Ald6 is a major source of the cytosolic NADPH.

A. Schematic highlighting that, per acetyl-CoA, two NADPH active hydrides and one proton from $\mathrm{H}_{2} \mathrm{O}$ are incorporated into newly synthesized fatty acids.

B. Cytosolic NADPH labeling (derived from fatty acid labeling, and corrected for substrate labeling fraction but not $\mathrm{H}$-D exchange or kinetic isotope effect) from [1,1-2 $\left.\mathrm{H}_{2}\right]$ ethanol in wild-type $\mathrm{S}$. cerevisiae and $\Delta z w f 1$ strain (mean, SE, $\mathrm{n}=6$ (3 biological replicates with results from both $\mathrm{C} 16: 0$ and $\mathrm{C} 18: 0$ ); ${ }^{* *}$, p<.001 by t-test).

C. Cytosolic NADPH labeling as in B from $\left[1{ }^{-2} \mathrm{H}\right]$ glucose in wild-type $S$. cerevisiae and $\Delta$ ald 6 strain (mean, SE, $n=6$ ( 3 biological replicates with results from both $C 16: 0$ and $C 18: 0) ; * * *, p<.001$ by $t$-test).

D. Summary of data from $B, C$, and $D_{2} \mathrm{O}$ tracing into fat shows sources of cytosolic NADPH redox active hydrogen nucleus in wild-type $S$. cerevisiae $F Y 4$ (mean, SE, $n=6$ ( 3 biological replicates with results from both $\mathrm{C} 16: 0$ and $\mathrm{C} 18: 0)$ ). The redox active hydrogen nucleus, but not the associated high energy electrons, is in a rapid H-D exchange with water, which explains the fractional contribution not accounted for by the pentose phosphate pathway and Ald6. 

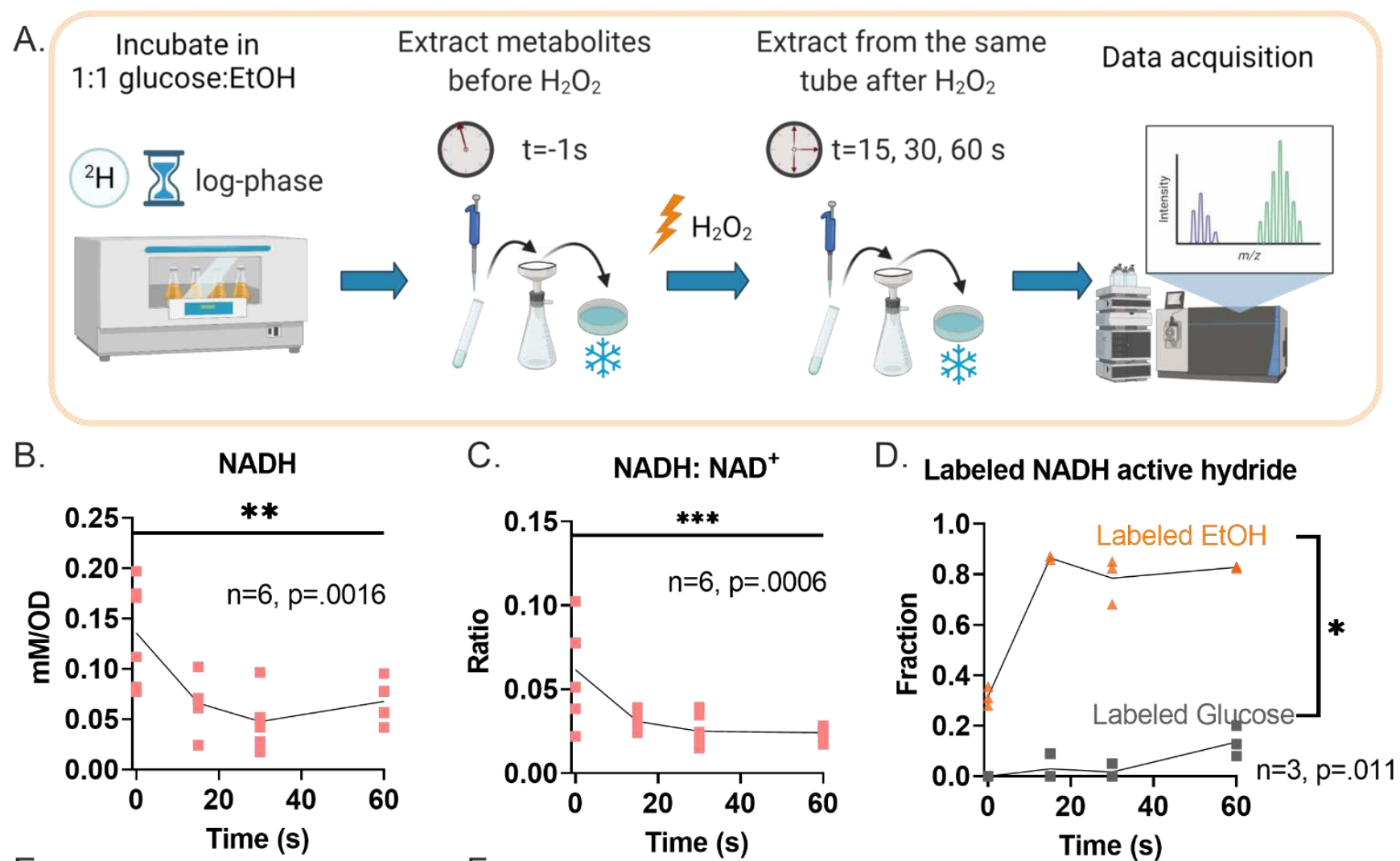

E.

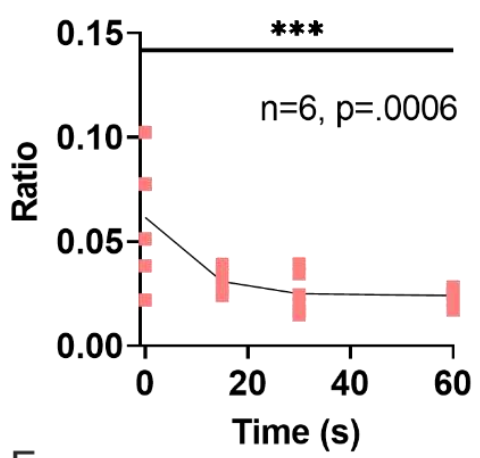

F. NADPH: NADP ${ }^{+}$

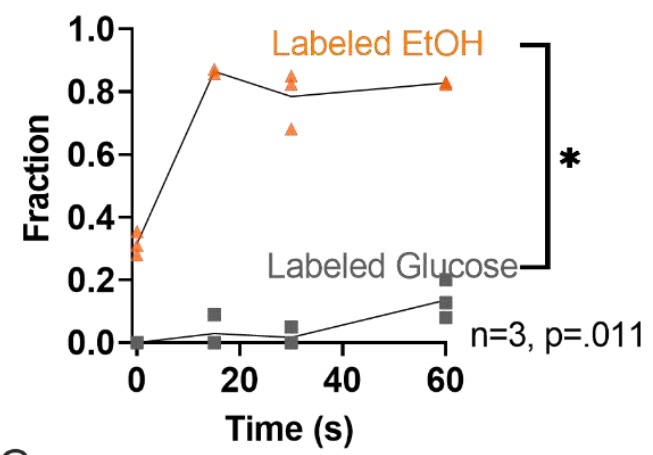

NADPH
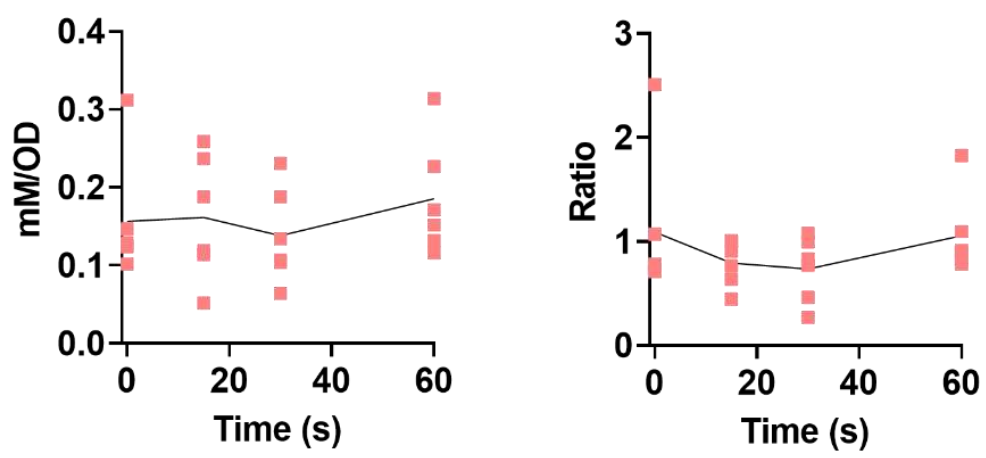

G. Labeled NADPH active hydride

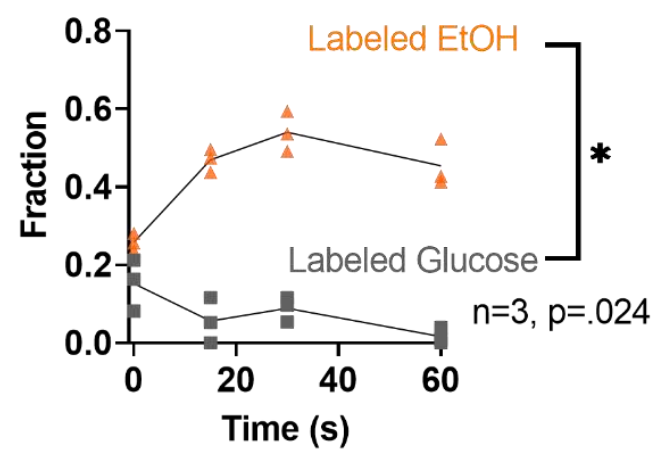

Figure 6. Instead of oxPPP, ethanol oxidation provides major fraction of active hydrides in the presence of hydrogen peroxide.

A. Schematic of experimental workflow of administration of $\mathrm{H}_{2} \mathrm{O}_{2}$ oxidative stress to yeast (S.cerevisae FY4) cultures pre-incubated with equicarbon glucose:ethanol (Created with Biorender). Temporal changes within the first $60 \mathrm{~s}$ after $\mathrm{H}_{2} \mathrm{O}_{2}$ shock are captured by rapid quenching of metabolism at time points of 15,30 , and $60 \mathrm{~s}$.

B. NADH pool size (mean, SE, $n=6$ (biological replicates), negative linear trend, $p=.0016$, ordinary oneway ANOVA).

C. NADH: NAD+ratio (mean, $\mathrm{SE}, \mathrm{n}=6$ (biological replicates), negative linear trend, $\mathrm{p}=.0006$, ordinary oneway ANOVA).

D. $\mathrm{NADH}$ active hydride labeling from $[1,1-2 \mathrm{H} 2]$ ethanol (orange) and $\left[1-{ }^{2} \mathrm{H}\right]$ glucose (grey) (mean, $\mathrm{SE}, \mathrm{n}=3$ (biological replicates), $p=.011\left(^{*}\right)$, two-tailed paired t-test). Increase in the NADH active hydride from labeled ethanol is statistically significant (positive linear trend, $p<0.001$, ordinary one-way ANOVA).

E. NADPH pool size (mean, SE, $n=6$ (biological replicates)).

F. NADPH: NADP+ ratio (mean, SEM, $n=6$ (biological replicates)).

G. NADPH active hydride labeling from $\left[1,1-{ }^{2} \mathrm{H}_{2}\right]$ ethanol (orange) and $[1-2 \mathrm{H}]$ glucose (grey) (mean, $\mathrm{SE}, \mathrm{n}=3$ (biological replicates), $p=.02$, two-tailed paired t-test). Increase in the NADPH active hydride labeled by ethanol is statistically significant (positive linear trend, $p<.001$, ordinary one-way ANOVA). 


\section{Supplementary Files}

This is a list of supplementary files associated with this preprint. Click to download.

- NCHEMBA211214119Supplementary.pdf

- JRabinowitzEPCflatnew.pdf 